\title{
Down's syndrome in diabetic patients aged $<20$ years: an analysis of metabolic status, glycaemic control and autoimmunity in comparison with type 1 diabetes
}

\author{
T. R. Rohrer • P. Hennes - A. Thon • A. Dost • \\ M. Grabert • B. Rami $\cdot$ S. Wiegand $\cdot$ R. W. Holl • \\ on behalf of the DPV Initiative
}

Received: 11 November 2009/Accepted: 20 January 2010/Published online: 26 February 2010

(C) Springer-Verlag 2010

\begin{abstract}
Aims/hypothesis Intellectual impairment in individuals with Down's syndrome and diabetes mellitus potentially limits the quality of diabetic control. In addition, these patients are at risk of having immunological abnormalities. The present study compared metabolic status and concomitant diseases in young $(<20$ years old) Down's syndrome patients with diabetes vs young type 1 diabetic patients.

Methods The Diabetes-Patienten-Verlaufsdaten is a longitudinal follow-up database, which collects data from 298 German and Austrian diabetes centres. Data available on diabetic patients aged $<20$ years were analysed statistically.
\end{abstract}

T. R. Rohrer $(\bowtie) \cdot$ P. Hennes

Department of Paediatrics and Neonatology,

Saarland University Hospital,

Kirrberger Str. 1, Geb. 9,

66421 Homburg/Saar, Germany

e-mail: tilman.rohrer@uniklinikum-saarland.de

A. Thon

Hannover Medical School, Children's Hospital,

Hannover, Germany

A. Dost

Department of Paediatrics, Friedrich Schiller University of Jena,

Jena, Germany

M. Grabert $\cdot$ R. W. Holl

Department of Epidemiology, University of Ulm,

Ulm, Germany

B. Rami

Department of Paediatrics, Medical University of Vienna,

Vienna, Austria

S. Wiegand

Institute of Experimental Paediatric Endocrinology,

Universitätsmedizin Berlin,

Berlin, Germany
Results We compared data for 159 Down's syndrome patients with diabetes and 41,983 type 1 diabetic patients. The former used less insulin, but showed better glycaemic control $\left(\mathrm{HbA}_{1 \mathrm{c}}\right)$. Diabetes onset during the first 3 years of life occurred in $18.9 \%$ of Down's syndrome patients with diabetes and in $6.4 \%$ of type 1 diabetic patients. Antibody titres indicative of coeliac disease and thyroid peroxidase antibodies were more frequent in Down's syndrome patients with diabetes. No significant differences were found regarding the beta cell autoantibodies studied.

Conclusions/interpretation The age-of-onset distribution showed a shift towards younger ages and was bimodal in the Down's syndrome group. The better metabolic control found, despite intellectual impairment, in young Down's syndrome patients with diabetes cannot be conclusively explained by our data, but is likely to be due to a less complex lifestyle. Our data provide further confirmation that coeliac and thyroid antibodies are more prevalent in Down's syndrome. The presence of beta cell autoantibodies supports an autoimmune cause of diabetes in some children with Down's syndrome.

Keywords Beta cell autoantibodies - Coeliac antibodies . Diabetes mellitus · Down's syndrome · Haemoglobin $A_{1 \mathrm{c}}$. Insulin · Islet autoantibodies · Metabolism · Thyroid · Trisomy 21
Abbreviations
ANCOVA Analysis of covariance
CSII Continuous subcutaneous insulin infusion
DPV Diabetes-Patienten-Verlaufsdaten (longitudinal follow-up data on diabetic patients)
GLIMMIX Generalised linear mixed models
IA-2A Tyrosine phosphatase IA-2 antibodies 


\section{Introduction}

Individuals with Down's syndrome (trisomy 21) are more likely to have diabetes mellitus than those without this genetic condition. Dutch researchers reported a threefold increase in the prevalence of type 1 and type 2 diabetes in patients with Down's syndrome compared with the general population [1]. In Denmark the prevalence of Down's syndrome among patients with diabetes is 4.2 times higher than in the general population [2].

In addition, Down's syndrome is associated with an increased prevalence of autoimmune disorders such as coeliac disease [3] and hypothyroidism [4].

The aim of the present study was to compare young (less than 20 years old) diabetic patients with Down's syndrome (assigned to type 3 diabetes mellitus according to the classification proposed by the WHO and the American Diabetes Association [5]) and without Down's syndrome (type 1 diabetes mellitus) in respect of various markers of autoimmune disease and diabetic control by using the extensive Diabetes-Patienten-Verlaufsdaten (DPV) database, a longitudinal follow-up database on diabetic patients, to which 298 German and Austrian diabetes centres regularly contribute long-term follow-up data.

A further aim was to investigate whether different forms of diabetes were distinguishable in Down's syndrome patients with diabetes. We differentiated according to the presence or absence of diabetes-associated autoantibodies in that group.

\section{Methods}

All patient data were collected during routine care. Each centre complied with local ethical and data management guidelines. The relevant Ethics Committees were informed of the DPV database and subsequent data analysis studies, and had no concerns. Data available from the DPV database as of April 2009 were included in the present analysis.

The SAS statistical software package (version 9.13; SAS Institute, Cary, NC, USA) was used to calculate means and standard deviations for a number of variables indicative of metabolic status, glycaemic control and autoimmunity. Data analysis was performed for relevant demographic and clinical variables on all diabetic Down's syndrome patients and non-Down's syndrome type 1 diabetic patients who were less than 20 years of age at the time of analysis. Patients with other, secondary forms of diabetes were excluded. Means \pm SD and percentages, where applicable, for the Down's syndrome group were compared statistically with those for the group of type 1 diabetic patients without Down's syndrome. Comparison was by means of the Kruskal-Wallis test. As multiple tests were performed, $p$ values were adjusted using the Bonferroni stepdown correction (Holm method) [6]. Down's syndrome patients with diabetes and type 1 diabetic patients were compared for rate of diabetes onset under the age of 3 years. The $\chi^{2}$ test was used to calculate the level of statistical significance. Glycaemic control was evaluated in terms of $\mathrm{HbA}_{1 \mathrm{c}}$ levels, which were determined using the method established at each individual centre. To adjust for differences between participating laboratories, the $\mathrm{HbA}_{1 \mathrm{c}}$ data were mathematically standardised according to the Diabetes Control and Complications Trial (DCCT) reference range of 4.05 $6.05 \%\left(\mathrm{HbA}_{1 \mathrm{c}}-\mathrm{DCCT}\right)$ using the multiple of the mean method [7].

A multiple regression analysis of covariance (ANCOVA) was used to identify the variables affecting the dependent variable $\mathrm{HbA}_{1 \mathrm{c}}$ in Down's syndrome patients with diabetes. The following independent variables were included in the analysis using the generalised linear mixed models procedure (PROC GLIMMIX; SAS version 9.13; SAS Institute, Cary, NC, USA): age at onset of diabetes, duration of diabetes, sex, migration background, BMI SD score, insulin regimen (number of injections per day), insulin dose and use of both slow- and fast-acting insulin analogues. The reporting centre was included in the model as a random variable to adjust for intra-centre correlation (hierarchical model).

Remission periods were defined as periods during which daily insulin requirements were $<0.5 \mathrm{U} / \mathrm{kg}$ and $\mathrm{HbA}_{1 \mathrm{c}}$ levels were $<7.5 \%$. Again, variables affecting the duration of remission as the dependent variable were identified by analysis of covariance (ANCOVA). The independent variables evaluated in this GLIMMIX analysis were: age at onset of diabetes, sex and migration background, with reporting centre as a random variable.

\section{Results}

At the time of analysis, the DPV database contained information on 159 individuals who met the criteria for the Down's syndrome group and on 41,983 individuals who met the criteria for the group of patients with type 1 diabetes.

The key demographic data and clinical characteristics of both groups are shown in Table 1. The two groups were comparable with respect to sex, age and both onset and duration of diabetes. There was a trend towards a higher BMI in the Down's syndrome group. Due to their primary condition, children and adolescents with Down's syndrome were of shorter stature. Height was $-2.7 \pm 1.2$ SD score in the 150 Down's syndrome patients with diabetes for whom height data were available, compared with $-0.05 \pm 1.0 \mathrm{SD}$ score in 40,386 type 1 diabetic patients $(p<0.001)$. Due to 
Table 1 Demographic data and clinical characteristics for young type 3 diabetic patients with Down's syndrome and young type 1 non-Down's syndrome diabetic patients

Unless otherwise indicated, values are means $\pm \mathrm{SD}$

The numbers of evaluable patients vary according to the availability of complete data sets for the various target variables

All patients were $<20$ years old

\begin{tabular}{llllc}
\hline Variable & Down's+diabetes & Type 1 diabetes only & $p$ value & $p$ value corrected \\
\hline All patients $(n)$ & 159 & 41983 & & \\
Male, $n(\%)$ & $86(54.1)$ & $21,999(52.4)$ & 0.67 & 1.0 \\
Female, $n(\%)$ & $73(45.9)$ & $19,984(47.6)$ & & \\
Age (years) & $14.35 \pm 5.23$ & $13.76 \pm 4.33$ & $<0.001$ & $<0.05$ \\
Patients $(n)$ & 159 & 41,983 & & \\
BMI & $22.59 \pm 5.66$ & $21.08 \pm 4.10$ & $<0.01$ & 0.1 \\
BMI SDS & $0.81 \pm 1.09$ & $0.51 \pm 0.96$ & $<0.01$ & \\
Patients $(n)$ & 150 & 40,261 & & \\
Diabetes age of onset (years) & $8.21 \pm 5.29$ & $8.42 \pm 4.26$ & 0.41 & \\
Patients $(n)$ & 159 & 41,983 & & \\
Diabetes duration (years) & $6.14 \pm 5.19$ & $5.34 \pm 4.18$ & 0.25 & \\
Patients $(n)$ & 159 & 41,983 & & $<0.001$ \\
Insulin injections daily & $3.73 \pm 1.91$ & $4.92 \pm 2.19$ & $<0.001$ & \\
Patients $(n)$ & 153 & 40,087 & & $<0.05$ \\
Insulin dose (U/kg) & $0.78 \pm 0.33$ & $0.85 \pm 0.31$ & & \\
Patients $(n)$ & 152 & 39,598 & & \\
\hline
\end{tabular}

their shorter height, body weight in the Down's syndrome patients with diabetes was $-0.85 \pm 1.29$ SD score vs $0.40 \pm$ 1.0 SD score $(p<0.001 ; n=154)$ and thus also lower than in the 40,595 type 1 diabetic patients. Analysis using an uncorrected $p$ value showed the BMI SD score to be significantly increased $(p<0.01)$, but the corrected $p$ value did not reach statistical significance $(p=0.1)$.

The ages of diabetes onset in Down's syndrome patients with diabetes and in type 1 diabetic patients were $8.2 \pm 5.3$ and $8.4 \pm 4.3$ years (mean \pm SD), respectively. This difference was not statistically significant. However, age distribution differed in that onset during the first 3 years of life occurred in $18.9 \%$ of Down's syndrome patients with diabetes, but only in $6.4 \%$ of type 1 diabetic patients $(p<0.0001)$. Moreover, age of diabetes onset showed two peaks for Down's syndrome patients with diabetes, whereas it was approximately Gaussian in shape for type 1 diabetic patients (Fig. 1).

Patients in the Down's syndrome group received fewer insulin injections per day and lower insulin doses. Data on insulin therapy were analysed for 159 Down's syndrome patients with diabetes, of whom $35.8 \%$ were receiving conventional treatment (twice daily mixed insulin regimen), $52.8 \%$ were on intensive conventional treatment (four times daily insulin regimen, split dose basal insulin replacement) and $5.7 \%$ were being treated by continuous subcutaneous insulin infusion (CSII). In contrast, 14\% of type 1 diabetic patients were on conventional therapy, $64 \%$ were on intensive conventional therapy and $15 \%$ were being treated by CSII. Significant differences in multiple step-down corrections were found for conventional therapy $(p<0.001)$, intensive conventional therapy $(p=0.08)$ and CSII $(p<0.05)$ in Down's syndrome patients with diabetes. Fast-acting insulin ana- logues were used more frequently by type 1 diabetic patients (48.9\% vs $25.7 \%, p<0.001$ ). Similarly, the use of longacting insulin analogues was significantly more frequent in type 1 diabetic patients than in Down's syndrome patients with diabetes $(39.8 \%$ vs $25.2 \%, p<0.05)$.

Glycaemic control, as measured by $\mathrm{HbA}_{1 \mathrm{c}}$ levels, was better in the group of Down's syndrome patients with diabetes than in the group of type 1 diabetic patients $(p<0.01)$.

Compared with type 1 diabetic counterparts, Down's syndrome patients with diabetes had a roughly twofold increase in the frequency of antibodies and/or autoantibodies related to coeliac disease and thyroid disease (Table 2).

There were no significant differences between the two groups with regard to diabetic complications such as nephropathy or retinopathy. Furthermore, no differences were observed in the manifestation of diabetic ketoacidosis at diagnosis or in the further progression of the disease. No difference was noted for the occurrence of severe hypoglycaemia or coma.

To distinguish different forms of diabetes in the Down's syndrome group, we looked for the presence of autoantibodies associated with type 1 diabetes (collectively referred to here as beta cell autoantibodies), including islet cell antibodies (ICA), GAD antibodies, insulin autoantibodies and tyrosine phosphatase IA-2 antibodies (IA-2A, Table 3). Data on beta cell autoantibody measurements were available for 56 Down's syndrome patients with diabetes. Of these, 13 had no autoantibodies vs 43 patients with diabetes-associated autoantibodies. The respective BMI SD scores of $0.71 \pm 1.04$ and $0.77 \pm 1.03$ for Down's syndrome patients with diabetes and type 1 diabetic patients were comparable. The insulin doses for antibody-negative 

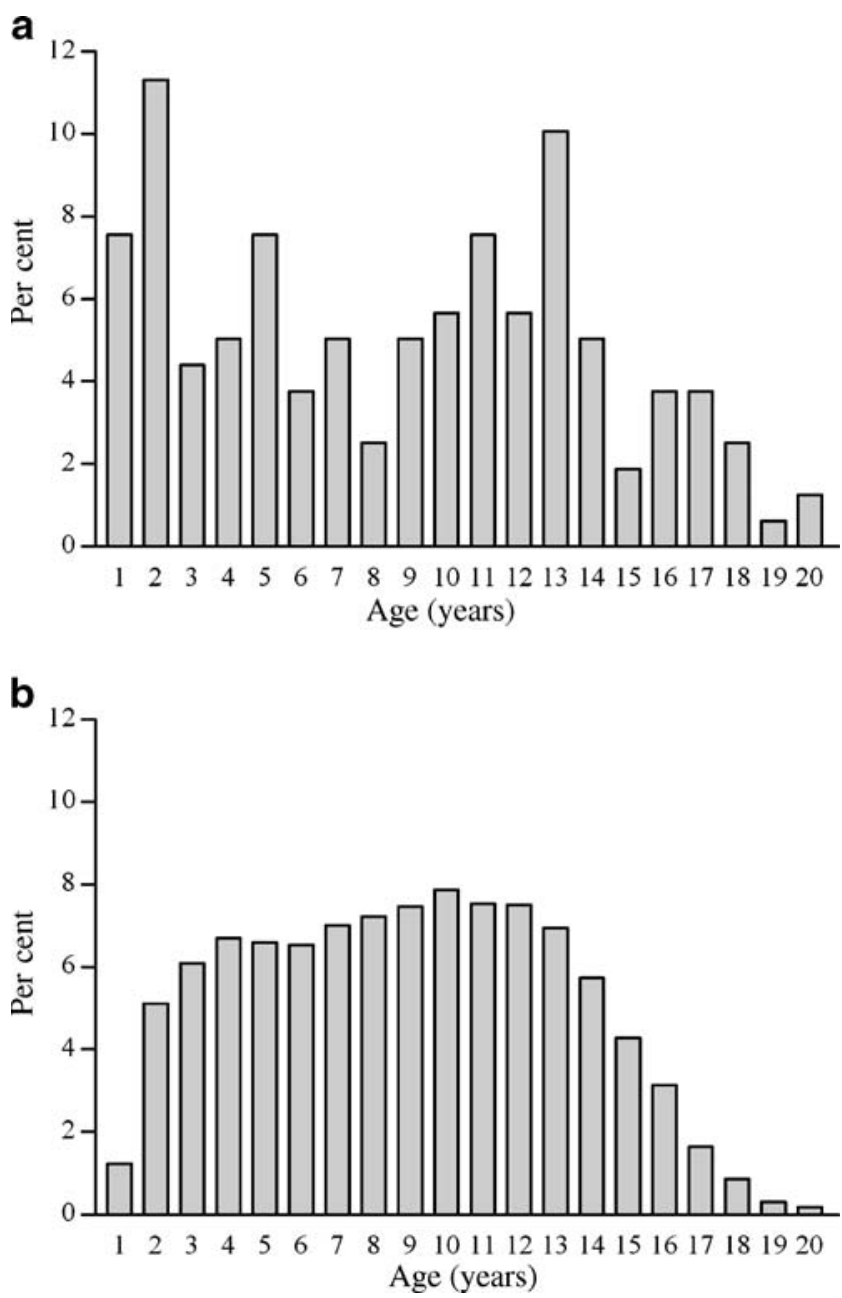

Fig. 1 Distribution of age of diabetes onset in (a) patients with Down's syndrome and diabetes mellitus and (b) type 1 diabetic patients

and antibody-positive Down's syndrome patients with diabetes were $0.78 \pm 0.23$ and $0.78 \pm 0.41 \mathrm{Ukg}^{-1} \mathrm{day}^{-1}$ (mean $\pm \mathrm{SD}$ ) respectively. Diabetic ketoacidosis and severe diabetic ketoacidosis with $\mathrm{pH}<7.1$ at the onset of diabetes occurred in none of the diabetic Down's syndrome patients who were antibody-negative. Of those who had detectable autoantibodies, $33 \%$ and $0 \%$ had had diabetic ketoacidosis and severe diabetic ketoacidosis, respectively, at diabetes onset.

In the multiple regression analysis using $\mathrm{HbA}_{1 \mathrm{c}}$ as the dependent variable in Down's syndrome patients with diabetes, the only detectable association was that for insulin dose, with a $\beta$ value of 1.23 , a standard error of $\beta=0.38$ and significance at $p<0.01$. A further multiple regression analysis showed no effect of age at diagnosis of diabetes, sex or migration background on the duration of remission in Down's syndrome patients with diabetes. There were no differences in insulin dose between boys and girls in the Down's syndrome group of patients with diabetes.
However, significant differences in associated autoimmunity were observed. In girls, both with or without Down's syndrome, autoimmune diseases of the thyroid and coeliac antibodies were markedly more frequent. Thyroid antibodies were found in $19 \%$ of girls and $11 \%$ of boys with type 1 diabetes, respectively (corrected $p<0.001$ ), whereas such antibodies were present in $40 \%$ of girls and $35 \%$ of boys in the Down's syndrome group of patients with diabetes, respectively (corrected $p=1.0$ ).

There were no significant differences between the Down's syndrome patients with diabetes and the group of type 1 diabetic patients with regard to islet autoantibodies (Table 3). The only exception was IA-2A, for which the corrected $p$ value indicated significantly lower levels in Down's syndrome patients with diabetes.

\section{Discussion}

We analysed data from 42,142 young diabetic patients aged less than 20 years, $159(0.38 \%$ or 38 per 10,000$)$ of whom had Down's syndrome. This is very close to the prevalence of $0.38 \%$ reported in a study of the Danish population [2] that suggested an association with HLA genotypes and the possibility of genetic risk loci on chromosome 21 . The $0.38 \%$ prevalence of Down's syndrome in our population of diabetic patients was much higher than the Down's syndrome prevalence in other general populations. For instance, a study from the Netherlands reported a prevalence of Down's syndrome of 16 per 10,000 live births [8]. The Dutch study also reported increased neonatal and infant mortality in patients with Down's syndrome, which would suggest an even lower prevalence in older children. This is in line with a prevalence of 13 per 10,000 of the general population of children and adolescents as reported from the USA [9].

The Danish study [2] found that the onset of type 1 diabetes occurred at an earlier age of 6 years in Down's syndrome patients compared with 8.2 years in the background population. In our study, we found a smaller difference of 8.2 vs 8.4 years. As the Danish study included only eight patients with Down's syndrome, as opposed to the 159 patients in our study, we are confident that our data reflect the true difference more accurately.

The different age distributions within our cohort may be indicative of different aetiological mechanisms. Analyses that included diabetes-associated antibodies yielded no relevant indications; however, data sets were not sufficiently complete to allow any conclusions about HLA genotypes or the occurrence of diabetes-specific antibodies. This may also be due to the retrospective analysis of data, some of which were collected at a time when some or all of these antibodies were not yet known. In an extensive study of 
Table 2 Immunological, endocrine and glycaemic control variables in young type 1 diabetic patients and type 3 diabetic patients with Down's syndrome

Unless otherwise indicated, values are means \pm SD

${ }^{\text {a }}$ Given as percentages of patients with detectable antibodies

${ }^{\mathrm{b}}$ Coeliac-specific antibodies (IgG and $\operatorname{IgA}$ antibodies to gliadin, endomysium or tissue transglutaminase IgA antibodies)

\begin{tabular}{|c|c|c|c|c|}
\hline Variable & Down's+diabetes & Type 1 diabetes only & $p$ value & $p$ value corrected \\
\hline Patient total $(n)$ & 159 & 41,983 & & \\
\hline $\mathrm{HbA}_{1 \mathrm{c}}(\%)$ & $7.8 \pm 1.4$ & $8.5 \pm 1.8$ & $<0.001$ & $<0.001$ \\
\hline $\mathrm{HbA}_{1 \mathrm{c}}-\mathrm{DCCT}$ & $7.6 \pm 1.4$ & $8.3 \pm 1.8$ & $<0.001$ & $<0.001$ \\
\hline Patients $(n)$ & 152 & 40,763 & & \\
\hline \multicolumn{5}{|l|}{ Antibodies $^{\mathrm{a}}$} \\
\hline Anti-thyroglobulin (\%) & 37.2 & 14.9 & $<0.001$ & $<0.001$ \\
\hline Patients $(n)$ & 86 & 18,952 & & \\
\hline Thyroxine peroxidase (\%) & 40.8 & 15.8 & $<0.001$ & $<0.001$ \\
\hline Patients $(n)$ & 103 & 24,890 & & \\
\hline Coeliac $^{\mathrm{b}}(\%)$ & 50.5 & 20.4 & $<0.001$ & $<0.001$ \\
\hline Patients $(n)$ & 95 & 22,277 & & \\
\hline Anti-gliadin IgA (\%) & 31.5 & 6.8 & $<0.001$ & $<0.001$ \\
\hline Patients $(n)$ & 73 & 17,255 & & \\
\hline
\end{tabular}

islet cell autoimmunity [10], the prevalence of diabetesassociated HLA class II genotypes was the same in diabetic patients with or without Down's syndrome. However, the frequency of the highest risk HLA class II genotypes in diabetic patients with Down's syndrome was half as high as in diabetic patients without Down's syndrome. The authors also suggested that there might be loci on chromosome 21 that enhance the penetrance of diabetogenic genes.

In our study, the frequencies of islet autoantibodies were similar in Down's syndrome patients with diabetes and in patients with type 1 diabetes. Therefore, in view of the classification proposed by the American Diabetes Association [5], it appears very likely that diabetes in young

Table 3 Islet autoantibodies in young type 1 diabetic patients and in young type 3 diabetic patients with Down's syndrome

\begin{tabular}{|c|c|c|c|c|}
\hline Variable & $\begin{array}{l}\text { Down's+ } \\
\text { diabetes }\end{array}$ & $\begin{array}{l}\text { Type } 1 \\
\text { diabetes only }\end{array}$ & $p$ value & $\begin{array}{l}p \text { value } \\
\text { corrected }\end{array}$ \\
\hline ICA $(\%$ of $n)$ & 40.0 & 58.1 & $<0.05$ & 0.6 \\
\hline Patients $(n)$ & 40 & 10,475 & & \\
\hline GADA $(\%$ of $n)$ & 66.0 & 65.7 & 0.97 & 1.0 \\
\hline Patients $(n)$ & 47 & 11,228 & & \\
\hline IAA $(\% \text { of } n)^{\mathrm{a}}$ & 63.3 & 54.7 & 0.57 & 1.0 \\
\hline Patients $(n)$ & 22 & 5,616 & & \\
\hline IA-2A $(\%$ of $n)$ & 35.3 & 66.9 & $<0.001$ & $<0.01$ \\
\hline Patients $(n)$ & 34 & 7,701 & & \\
\hline $\begin{array}{l}\text { Beta cell } \\
\text { autoantibodies } \\
(\% \text { of } n)\end{array}$ & 76.8 & 82.0 & 0.31 & 1.0 \\
\hline Patients $(n)$ & 56 & 14,813 & & \\
\hline
\end{tabular}

Values are given as $n$ or percentages of patients with detectable antibodies

${ }^{a}$ During first 4 weeks after diagnosis

IAA, insulin autoantibodies; ICA, islet cell antibodies; GADA, GAD antibodies
Down's syndrome patients is of the autoimmune type. Our data are in agreement with a study from the UK, which reported a higher frequency of subclinical islet autoimmunity in children with Down's syndrome [10].

Our data show that there were no differences between autoantibody-positive and autoantibody-negative young Down's syndrome patients with diabetes with respect to daily insulin dose, BMI SD score or the occurrence of diabetic ketoacidosis at the onset of diabetes. This also suggests that type 3 diabetes in children and adolescents with Down's syndrome should be viewed in the context of increased autoimmunity in type 1 diabetes mellitus.

In spite of their intellectual impairment, Down's syndrome patients had better metabolic control of their diabetes than the group of type 1 diabetic patients, as judged by $\mathrm{HbA}_{1 \mathrm{c}}$ levels. They appeared to have simpler insulin treatment regimens, with fewer injections per day $(p<$ 0.001 ) and a trend towards lower doses. Our data are in agreement with the findings of a study from Scotland, which also reported better glycaemic control and lower insulin doses in Down's syndrome patients with diabetes [11]. In contrast, a study from Brazil observed insulin resistance (without manifest diabetes) in overweight adolescent girls with Down's syndrome [12]. This was not confirmed by our data. The slightly higher insulin doses in our Down's syndrome group relative to adults with Down's syndrome may be explained by the increased insulin requirement during growth and puberty, whereas the Scottish study was conducted in adult diabetes patients with Down's syndrome [11].

The simpler lifestyle and acceptance of routine could explain the better metabolic control in diabetic patients with Down's syndrome [11]. It has also been suggested that diabetic patients with Down's syndrome have a lower prevalence of diabetic retinopathy [13]. This could be due to better metabolic control or a lower risk HLA genotype 
[10]. In our study cohort, microalbuminuria was found in $3.1 \%$ of 65 young Down's syndrome patients with diabetes after a mean of 6.1 years of diabetes duration. This compares with microalbuminuria being present in $17.0 \%$ of 24,948 young type 1 diabetic patients after 5.3 years. The Scottish study reported complications in the form of nephropathy and neuropathy in two out of 13 Down's syndrome patients with diabetes, one of whom had endstage renal disease [11]. Our cohort included no diabetic Down's syndrome patients with diabetic retinopathy, which was present in $2.1 \%$ of 21,732 type 1 diabetic patients. This is in agreement with data from Ireland showing a lower prevalence of diabetic retinopathy in Down's syndrome patients with diabetes. The authors of that study hypothesised that lower blood pressure and lower IGF-1 levels in Down's syndrome patients with diabetes possibly had a protective effect [13].

Compared with people without Down's syndrome, Down's syndrome patients generally have significantly elevated risks of coeliac disease and hypothyroidism, which have been reported to be 4.7 and 9.4 times higher, respectively [4]. Our study provided additional evidence in support of these findings. In the Down's syndrome group, coeliac antibodies were increased twofold and gliadin IgA antibodies almost fivefold. We also observed a twofold increase in antithyroglobulin and thyroxine peroxidase antibodies in our Down's syndrome patients with diabetes.

In conclusion, the results of our analysis of the current DPV data confirm that diabetes mellitus in young individuals with Down's syndrome is more often due to autoimmunity than to insulin resistance and that additional comorbidities are more frequent in diabetic Down's syndrome patients than in non-Down's syndrome patients with type 1 diabetes. However, glycaemic control is better in the former group than in non-Down's syndrome type 1 diabetic patients, and that despite lower insulin doses. More specific studies are needed to identify the genetic factors that cause diabetes mellitus in Down's syndrome, e.g. the effects of the additional chromosome 21 or of alterations to the HLA genes on chromosome 6.

Acknowledgements The DPV programme was supported by the German Federal Ministry of Health, the German Diabetes Society (DDG), the Dr. Bürger-Büsing Foundation, the German Research Foundation (DFG), the National Action Forum on Diabetes Mellitus (NAFDM), the German Medical Association (Bundesärztekammer), the Kompetenznetz Diabetes mellitus (Competence Network for Diabetes mellitus; funded by the Federal Ministry of Education and
Research [FKZ 01GI0859]), the European Foundation for the Study of Diabetes (EFSD) and Novo Nordisk Pharma, Germany. The authors wish to acknowledge all patients, diabetes centres, investigators and staff who participated in the DPV initiative. A full listing of the participating centres has recently been published elsewhere [14].

Duality of interest statement The authors declare that there is no duality of interest associated with this manuscript.

\section{References}

1. Van Goor JC, Massa GG, Hirasing R (1997) Increased incidence and prevalence of diabetes mellitus in Down's syndrome. Arch Dis Child 77:186

2. Bergholdt R, Eising S, Nerup J, Pociot F (2006) Increased prevalence of Down's syndrome in individuals with type 1 diabetes in Denmark: a nationwide population-based study. Diabetologia 49:1179-1182

3. Bonamico M, Mariani P, Danesi HM et al (2001) Prevalence and clinical picture of celiac disease in Italian down syndrome patients: a multicenter study. J Pediatr Gastroenterol Nutr 33:139-143

4. Goldacre MJ, Wotton CJ, Seagroatt V, Yeates D (2004) Cancers and immune related diseases associated with Down's syndrome: a record linkage study. Arch Dis Child 89:1014-1017

5. American Diabetes Association (2009) Diagnosis and classification of diabetes mellitus. Diabetes Care 32(Suppl 1):S62-S67

6. Dmitrienko A, Molenberghs G, Chuang-Stein C, Offen W (2005) Multiple comparisons and multiple endpoints. In: Analysis of clinical trials using SAS. SAS Press, Cary, pp 67-128

7. The Diabetes Control and Complications Trial Research Group (1993) The effect of intensive treatment of diabetes on the development and progression of long-term complications in insulin-dependent diabetes mellitus. N Engl J Med 329:977-986

8. Weijerman ME, van Furth AM, Vonk NA, van Wouwe JP, Broers CJ, Gemke RJ (2008) Prevalence, neonatal characteristics, and first-year mortality of Down syndrome: a national study. J Pediatr 152:15-19

9. Besser LM, Shin M, Kucik JE, Correa A (2007) Prevalence of down syndrome among children and adolescents in metropolitan Atlanta. Birth Defects Res A Clin Mol Teratol 79:765-774

10. Gillespie KM, Dix RJ, Williams AJ et al (2006) Islet autoimmunity in children with Down's syndrome. Diabetes 55:3185-3188

11. Anwar AJ, Walker JD, Frier BM (1998) Type 1 diabetes mellitus and Down's syndrome: prevalence, management and diabetic complications. Diabet Med 15:160-163

12. Fonseca CT, Amaral DM, Ribeiro MG, Beserra IC, Guimaraes MM (2005) Insulin resistance in adolescents with Down syndrome: a cross-sectional study. BMC Endocr Disord 5:6

13. Fulcher T, Griffin M, Crowley S, Firth R, Acheson R, O'Meara N (1998) Diabetic retinopathy in Down's syndrome. Br J Ophthalmol 82:407-409

14. Schober E, Rami B, Grabert M et al (2009) Phenotypical aspects of maturity-onset diabetes of the young (MODY diabetes) in comparison with type 2 diabetes mellitus (T2DM) in children and adolescents: experience from a large multicentre database. Diabet Med 26:466-473 